\title{
Prediction of Color Properties of Cellulase-Treated 100\% Cotton Denim Fabric
}

\author{
C. W. Kan, W. Y. Wong, L. J. Song, and M. C. Law \\ Institute of Textiles and Clothing, The Hong Kong Polytechnic University, Hung Hom, Kowloon, Hong Kong \\ Correspondence should be addressed to C.W. Kan; tccwk@polyu.edu.hk
}

Received 13 November 2012; Revised 21 January 2013; Accepted 5 February 2013

Academic Editor: Jiri Militky

Copyright (C) 2013 C. W. Kan et al. This is an open access article distributed under the Creative Commons Attribution License, which permits unrestricted use, distribution, and reproduction in any medium, provided the original work is properly cited.

Artificial neural network (ANN) model was used for predicting colour properties of $100 \%$ cotton denim fabrics, including colour yield (in terms of K/S value) and CIE $L^{*}, a^{*}, b^{*}, C^{*}$, and $\mathbf{h}^{\circ}$ values, under the influence of cellulase treatment with various combinations of cellulase processing parameters. Variables examined in the ANN model included treatment temperature, treatment time, $\mathrm{pH}$, mechanical agitation, and fabric yarn twist level. The ANN model was compared with a linear regression model where the ANN model produced superior results in the prediction of colour properties of cellulase-treated $100 \%$ cotton denim fabrics. The relative importance of the examined factors influencing colour properties was also investigated. The analysis revealed that cellulase treatment processing parameters played an important role in affecting the colour properties of the treated $100 \%$ denim cotton fabrics.

\section{Introduction}

Denim jeans provide durability and vintage look for fashionable appearance which is the reason why they are welcomed by most people over the world. Conventional technologies involve creating designs by fading the colour of fabric by making the use of enzymatic treatment and bleach washing. Among these technologies, enzymatic treatment using cellulase is a commonly used method to achieve good colour fading effect with good fabric softness for $100 \%$ cotton denim fabrics. Although this technology could produce the desirable colour effects, there are a number side effects such as (i) difficulty in application, (ii) time consuming in processing, (iii) difficulty in creating standard and reproducible designs, (iv) effect cannot be applied to all textile surfaces, and (v) loss of quality if the process is not carefully controlled $[1,2]$. The literature review shows that artificial neural network (ANN) model has been used in many engineering fields [3-5]. In the case of the textile industry, ANN model is mainly used in yarn and fabric technologies [6-10]; no comprehensive ANN model has been used for predicting colour properties of $100 \%$ cotton denim material after cellulase treatment. Therefore, in this paper, we use artificial neural network (ANN) model for prediction of colour properties, including colour yield (in terms of K/S value) and CIE $L^{*}, a^{*}, b^{*}, C^{*}$, and $\mathbf{h}^{\circ}$ values of $100 \%$ cotton denim fabrics under the influence of cellulase treatment with the consideration of variables such as treatment temperature, treatment time, $\mathrm{pH}$, mechanical agitation, and fabric yarn twist level.

\section{Experimental}

2.1. Material. In this study, $100 \%$ desized $3 / 1$ right hand twill cotton denim fabrics were used, and their specifications were shown in Table 1.

2.2. Cellulase Treatment. A neutral cellulase enzyme (Lava Cell NNM, supplier: DyStar) in powder form was used for treating the denim fabric. Denim fabric samples (size of $22 \mathrm{~cm} \times 22 \mathrm{~cm}$ ) were treated with $0.1 \%$ cellulase with a liquor ratio of $50: 1$. Different cellulase treatments were conducted according to the different combination of treatment temperature $\left(50^{\circ} \mathrm{C}, 55^{\circ} \mathrm{C}\right.$, and $\left.60^{\circ} \mathrm{C}\right)$, treatment time $(30$ minutes, 45 minutes, 60 minutes, 75 minutes, and 90 minutes), mechanical agitation (simulated by 0,50 , and 100 steel balls for no mechanical agitation, mild mechanical agitation and severe mechanical agitation, resp.), and $\mathrm{pH}$ value $(6,7$, and 8). After cellulase treatment, the denim fabrics were rinsed with deionised water to lower the temperature quickly and 
TABLE 1: Specifications of denim fabrics.

\begin{tabular}{|c|c|c|c|c|c|c|}
\hline Fabric & 1 & 2 & 3 & 4 & 5 & 6 \\
\hline Fabric weight $\left(\mathrm{g} / \mathrm{m}^{2}\right)$ & $378 \pm 2.0$ & $380 \pm 1.8$ & $407 \pm 2.1$ & $400 \pm 2.0$ & $170 \pm 1.8$ & $178 \pm 1.7$ \\
\hline \multicolumn{7}{|l|}{ Density } \\
\hline Warp (ends/cm) & 25 & 25 & 26 & 26 & 27 & 27 \\
\hline Weft (picks/cm) & 20 & 20 & 18 & 19 & 20 & 20 \\
\hline \multicolumn{7}{|l|}{ Yarn count } \\
\hline Warp (Tex) & 88 & 88 & 88 & 88 & 88 & 88 \\
\hline Weft (Tex) & 57 & 57 & 57 & 57 & 57 & 57 \\
\hline Number of warp twists (per cm) & 4.3 & 3.5 & 4.7 & 3.9 & 4.2 & 3.7 \\
\hline Number of weft twists (per cm) & 4.7 & 4.7 & 4.7 & 4.7 & 4.7 & 4.7 \\
\hline
\end{tabular}

to wash away abraded substances attached on fabrics. The denim fabrics were then treated with deionised water at $80^{\circ} \mathrm{C}$ to deactivate the enzyme action. After that, the denim fabrics were squeezed to remove excessive water and were dried in an oven at a temperature of $70^{\circ} \mathrm{C}$ for two hours. Then, the denim fabrics were placed for conditioning under standard atmospheric pressure at $65 \pm 2 \%$ relative humidity and temperature of $20 \pm 2^{\circ} \mathrm{C}$ for at least 24 hours before further tests.

2.3. Colour Measurement. Colour properties were measured by a spectrophotometer of GretagMacbeth Color-Eye 7000A with D65 daylight and $10^{\circ}$ standard observer. Four measurements were taken for each fabric sample with $10 \mathrm{~mm}$ aperture and specular-excluded mode. The fabric samples were conditioned at $20 \pm 2{ }^{\circ} \mathrm{C}$ and relative humidity of $65 \pm 2 \%$ before taking the measurements. K/S sum in terms of summation of individual K/S value over the wavelength from $400 \mathrm{~nm}$ to $700 \mathrm{~nm}$ was calculated, and K/S sum value can help to determine the colour yield of the fabric samples. In addition, the CIE $\mathrm{L}^{*}, \mathrm{a}^{*}, \mathrm{~b}^{*}, \mathbf{C}^{*}$, and $\mathbf{h}^{\circ}$ values were also obtained which represents lightness/darkness, redness/greenness, yellowness/blueness, chroma, and hue, respectively.

2.4. Artificial Neural Network (ANN) Model. After cellulase treatment, six color-related data, that is, K/S sum value (color-ks), CIE L ${ }^{*}$ value (color-1), CIE a* value (color-a), $\mathrm{CIE} \mathrm{b}^{*}$ value (color-b), CIE $\mathbf{C}^{*}$ value (color-c), and CIE $\mathbf{h}^{\circ}$ (color-h) value, were obtained. In order to find the optimal neural network, ANN models having different topologies were formulated. The basic topology of the network is shown in Figure 1. In the ANN model, cellulase treatment processing factors, including treatment temperature (temperature), treatment time (time), $\mathrm{pH}(\mathrm{pH})$, and mechanical agitation (MA) were put in the input layer. Besides, fabric yarn twist level (twist) was also an important input node in case of cellulase-treated samples. For the output layer, the six nodes were corresponding to color-ks, color-l, color-a, color-b, color-c and color-h.

In order to train the ANN model, a typical three-layer network with Bayesian regulation backpropagation was used. It is considered as the most popular learning algorithm for the learning of a multilayered feed forward neural network

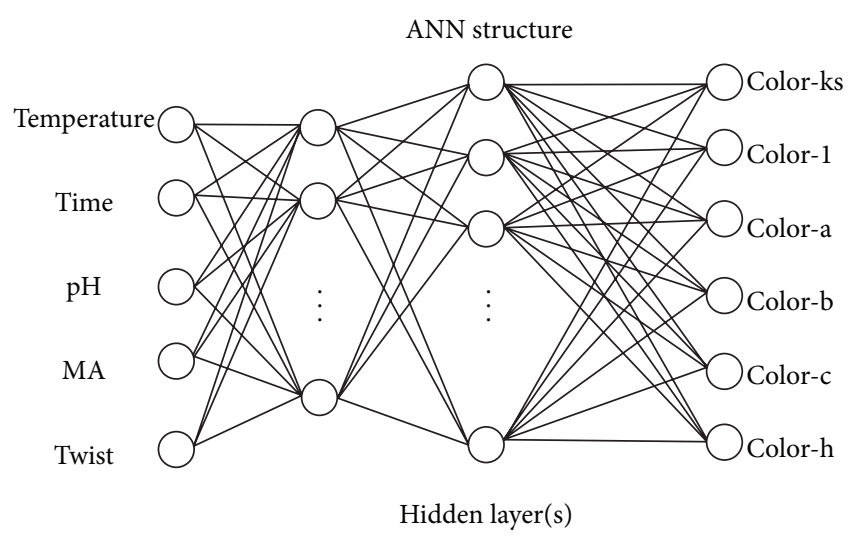

Figure 1: Basic structure of ANN model.

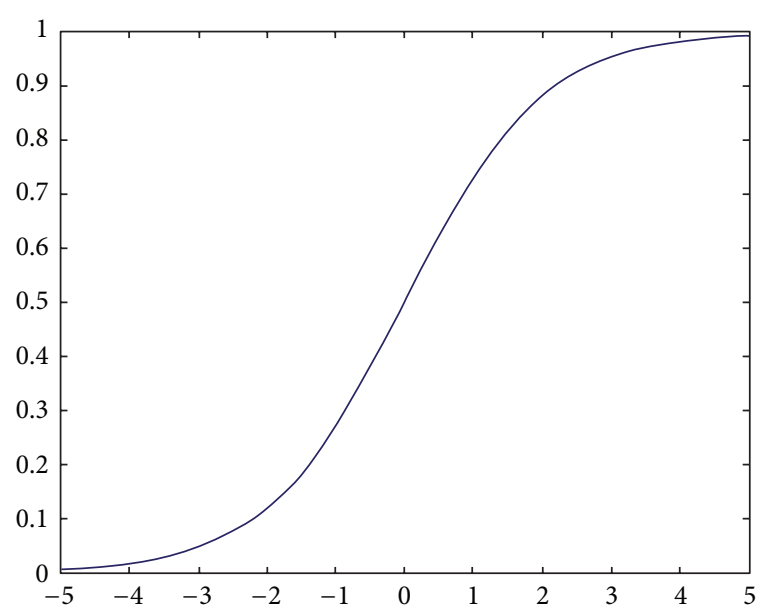

FIgURE 2: Sigmoid function.

[11]. For activation function, the sigmoid function is typically used [12], and in this case, was given by

$$
\operatorname{sigmoid}(a)=\frac{1}{1+e^{-a}} \text {. }
$$

This function can range between 0 and 1 and is differentiable. Figure 2 illustrates the function. Other parameters of the network were set as shown in Table 2. 
TABLE 2: Artificial neural network parameters.

\begin{tabular}{lcl}
\hline Parameter name & Value & Description \\
\hline net.trainParam.epochs & 1000 & Maximum number of epochs to train \\
net.trainParam.goal & $1 e-6$ & Performance goal \\
net.trainParam.mu & 0.005 & Marquardt adjustment parameter \\
net.trainParam.mu_dec & 0.1 & Decrease factor for mu \\
net.trainParam.mu_inc & 10 & Increase factor for mu \\
net.trainParam.mu_max & $1 e-10$ & Maximum value for mu \\
net.trainParam.max_fail & 10 & Maximum validation failures \\
net.trainParam.min_grad & $1 e-10$ & Minimum performance gradient \\
\hline
\end{tabular}

TABLE 3: Artificial neural network topology.

\begin{tabular}{lcccccccc}
\hline & \multicolumn{9}{c}{ Topology } & N6 & N7 & N8 & N9 \\
Number & N1 & N2 & N3 & N4 & N5 & N6 & 2 & 2 \\
Hidden layer & 1 & 1 & 1 & 1 & 1 & 2 & $10-10$ & $15-10$ \\
Nodes & 10 & 15 & 20 & 25 & 30 & $10-5$ & $20-10$ \\
\hline
\end{tabular}

TABLE 4: ANN statistical data for color-ks prediction.

\begin{tabular}{|c|c|c|c|c|c|c|c|c|c|}
\hline Network number & N1 & $\mathrm{N} 2$ & N3 & N4 & N5 & N6 & N7 & N8 & N9 \\
\hline \multicolumn{10}{|l|}{ MSE for different sets } \\
\hline Train error & 0.2926 & 0.0174 & 0.2721 & 0.9069 & 0.0351 & 0.0001 & 0.0001 & 0.0001 & 0.0001 \\
\hline Validate error & 21.394 & 18.295 & 8.945 & 12.862 & 4.991 & 0.011 & 0.003 & 0.011 & 0.005 \\
\hline Test error & 14.059 & 7.058 & 11.220 & 16.309 & 5.785 & 0.007 & 0.004 & 0.019 & 0.016 \\
\hline \multicolumn{10}{|l|}{ For total data } \\
\hline MAE & 1.1736 & 1.1202 & 1.1968 & 1.5670 & 0.7956 & 0.0309 & 0.0235 & 0.0383 & 0.0332 \\
\hline MSE & 7.1800 & 5.0185 & 4.1479 & 6.3110 & 2.1497 & 0.0037 & 0.0015 & 0.0060 & 0.0043 \\
\hline RMSE & 2.6796 & 2.2402 & 2.0366 & 2.5122 & 1.4662 & 0.0610 & 0.0381 & 0.0776 & 0.0653 \\
\hline Minimum error & 0.0017 & 0.0010 & 0.0043 & 0.0110 & 0.0009 & 0.0001 & 0.0002 & $8.7 E-05$ & 0.0001 \\
\hline Maximum error & 22.295 & 10.227 & 10.001 & 12.494 & 7.508 & 0.322 & 0.162 & 0.503 & 0.431 \\
\hline \multicolumn{10}{|c|}{ Relative error distribution } \\
\hline $0-1 \%$ & 155 & 153 & 157 & 152 & 160 & 162 & 162 & 162 & 162 \\
\hline $1 \%-5 \%$ & 7 & 9 & 5 & 10 & 2 & 0 & 0 & 0 & 0 \\
\hline $5 \%-10 \%$ & 0 & 0 & 0 & 0 & 0 & 0 & 0 & 0 & 0 \\
\hline $10 \%-50 \%$ & 0 & 0 & 0 & 0 & 0 & 0 & 0 & 0 & 0 \\
\hline$>50 \%$ & 0 & 0 & 0 & 0 & 0 & 0 & 0 & 0 & 0 \\
\hline
\end{tabular}

Network number 7 gives the best performance in the ANN models related to color-ks prediction with the smallest MSE of 0.0015.

In addition, determining the number of hidden layers and the number of nodes in each layer is not straightforward and still has not been solved perfectly. There are a number of theoretical results concerning the number of hidden layers in a network. Specifically, Hetcht-Nielsen has shown that a network with two hidden layers can approximate any arbitrary nonlinear function and generate any complex decision region for classification problems [12]. Later, it was shown by Cybenko that a single layer is enough to form an arbitrarily close approximation to any nonlinear decision boundary [13] . Hornik and Stinchombe have come up with a more general theoretical result. They have shown that a single hidden layer feed forward network with arbitrary sigmoid hidden layer activation functions can well approximate an arbitrary mapping from one finite dimensional space to another [11].
Generally speaking, with more nodes in hidden layer, the network's ability of approximation is increased while the generalization ability is decreased. Therefore, the structure of the ANN is usually decided by experience together with trials [14]. On top of that, the size of the training set and the number of input/output nodes also affect the topology of the optimal neural network [15].

In this study, nine different topologies of the neural network were constructed and tested. According to the experience and practice, considering the fact that the data size and the number of input/output nodes are small, ANN with more than 30 nodes in the hidden layer is not necessary in this case. This is because more nodes cannot get better performance and a lot of training time will be spent, which may be computationally wasteful. Smaller networks require 
TABLE 5: ANN statistical data for color-l prediction.

\begin{tabular}{|c|c|c|c|c|c|c|c|c|c|}
\hline Network number & N1 & $\mathrm{N} 2$ & N3 & N4 & N5 & N6 & N7 & N8 & N9 \\
\hline \multicolumn{10}{|l|}{ MSE for different sets } \\
\hline Train error & $1.9 E-04$ & $2.3 E-05$ & $1.1 E-04$ & $9.8 E-06$ & $1.8 E-04$ & $3.8 E-08$ & $3.9 E-08$ & $2.6 E-08$ & $3.5 E-08$ \\
\hline Validate error & 0.006 & 0.004 & 0.002 & 0.002 & 0.004 & $3.4 E-05$ & $9.7 E-05$ & $6.2 E-05$ & $6.8 E-05$ \\
\hline Test error & 0.003 & 0.002 & 0.005 & 0.005 & 0.004 & $1.6 E-05$ & $4.9 E-05$ & $5.6 E-05$ & $6.3 E-05$ \\
\hline \multicolumn{10}{|l|}{ For total data } \\
\hline MAE & 0.025 & 0.018 & 0.022 & 0.018 & 0.025 & 0.002 & 0.002 & 0.002 & 0.003 \\
\hline MSE & 0.002 & 0.001 & 0.001 & 0.001 & 0.002 & $9.9 E-06$ & $2.9 E-05$ & $2.3 E-05$ & $2.6 E-05$ \\
\hline RMSE & 0.0422 & 0.0346 & 0.0383 & 0.0368 & 0.0402 & 0.0031 & 0.0054 & 0.0048 & 0.0051 \\
\hline Minimum error & $2.0 E-05$ & $4.8 E-05$ & $4.4 E-05$ & $1.0 E-05$ & $4.2 E-05$ & $3.0 E-07$ & $2.0 E-06$ & $3.4 E-07$ & $1.6 E-06$ \\
\hline Maximum error & 0.231 & 0.154 & 0.218 & 0.205 & 0.177 & 0.014 & 0.041 & 0.021 & 0.024 \\
\hline \multicolumn{10}{|c|}{ Relative error distribution } \\
\hline $0-1 \%$ & 160 & 161 & 160 & 161 & 161 & 162 & 162 & 162 & 162 \\
\hline $1 \%-5 \%$ & 2 & 1 & 2 & 1 & 1 & 0 & 0 & 0 & 0 \\
\hline $5 \%-10 \%$ & 0 & 0 & 0 & 0 & 0 & 0 & 0 & 0 & 0 \\
\hline $10 \%-50 \%$ & 0 & 0 & 0 & 0 & 0 & 0 & 0 & 0 & 0 \\
\hline$>50 \%$ & 0 & 0 & 0 & 0 & 0 & 0 & 0 & 0 & 0 \\
\hline
\end{tabular}

Network number 6 gives the best performance in the ANN models related to color-1 prediction with the smallest MSE of 9.9E - 06.

TABLE 6: ANN statistical data for color-a prediction.

\begin{tabular}{lccccccccc}
\hline Network number & $\mathrm{N} 1$ & $\mathrm{~N} 2$ & $\mathrm{~N} 3$ & $\mathrm{~N} 4$ & $\mathrm{~N} 5$ & $\mathrm{~N} 6$ & $\mathrm{~N} 7$ & $\mathrm{~N} 8$ & $\mathrm{~N} 9$ \\
\hline $\begin{array}{l}\text { MSE for different sets } \\
\text { Train error }\end{array}$ & $3.0 E-09$ & $2.7 E-10$ & $1.1 E-09$ & $1.1 E-10$ & $3.8 E-10$ & $1.4 E-10$ & $3.6 E-10$ & $3.4 E-10$ & $1.2 E-10$ \\
$\quad$ Validate error & $2.5 E-08$ & $1.4 E-08$ & $1.5 E-08$ & $1.4 E-08$ & $1.6 E-08$ & $5.3 E-09$ & $1.1 E-08$ & $6.4 E-09$ & $7.3 E-09$ \\
$\quad$ Test error & $2.4 E-08$ & $1.9 E-08$ & $1.3 E-08$ & $3.4 E-08$ & $1.5 E-08$ & $1.2 E-08$ & $4.6 E-09$ & $6.9 E-09$ & $7.3 E-09$ \\
For total data & & & & & & & & & \\
MAE & $7.5 E-05$ & $4.1 E-05$ & $5.2 E-05$ & $4.8 E-05$ & $4.7 E-05$ & $3.5 E-05$ & $3.6 E-05$ & $3.5 E-05$ & $3.1 E-05$ \\
MSE & $1.2 E-08$ & $6.5 E-09$ & $6.2 E-09$ & $9.5 E-09$ & $6.4 E-09$ & $3.6 E-09$ & $3.3 E-09$ & $2.8 E-09$ & $3.0 E-09$ \\
RMSE & 0.0001 & $8.1 E-05$ & $7.9 E-05$ & $9.8 E-05$ & $8.0 E-05$ & $6.0 E-05$ & $5.7 E-05$ & $5.3 E-05$ & $5.4 E-05$ \\
Minimum error & $3.1 E-07$ & $1.4 E-07$ & $4.3 E-07$ & $4.8 E-08$ & $3.2 E-07$ & $1.5 E-07$ & $4.8 E-07$ & $9.7 E-08$ & $3.4 E-08$ \\
Maximum error & 0.0004 & 0.0006 & 0.0004 & 0.0005 & 0.0004 & 0.0002 & 0.0002 & 0.0002 & 0.0002 \\
Relative error distribution & & & & & & 162 & 162 & 162 & 162 \\
$0-1 \%$ & 162 & 162 & 162 & 162 & 162 & 162 \\
$1 \%-5 \%$ & 0 & 0 & 0 & 0 & 0 & 0 & 0 & 0 \\
$5 \%-10 \%$ & 0 & 0 & 0 & 0 & 0 & 0 & 0 & 0 \\
$10 \%-50 \%$ & 0 & 0 & 0 & 0 & 0 & 0 & 0 & 0 \\
$>50 \%$ & 0 & 0 & 0 & 0 & 0 & 0 & 0 & 0
\end{tabular}

Network number 8 gives the best performance in the ANN models related to color-a prediction with the smallest MSE of $2.8 E-09$.

less memory to store the connection weights and can be implemented in hardware easier and more economically. Training a smaller network usually requires less computation because each iteration is computationally less expensive. Smaller networks have also very short propagation delays from their inputs to their outputs. This is very important during the testing phase of the network, where fast responses are usually required. The details of the ANN topology are shown in Table 3.
In the experiment, there were totally 810 datasets for each group. These data were randomly divided into three sets: training (60\%), cross-validation $(20 \%)$, and test $(20 \%)$ in order to meet the requirements of both accuracy and generalization.

\section{Results and Discussion}

3.1. ANN Model. In order to evaluate the effect of ANN model, the MAE (mean absolute error), MSE (mean square 
TABLE 7: ANN statistical data for color-b prediction.

\begin{tabular}{|c|c|c|c|c|c|c|c|c|c|}
\hline Network number & N1 & $\mathrm{N} 2$ & $\mathrm{~N} 3$ & $\mathrm{~N} 4$ & N5 & N6 & N7 & N8 & N9 \\
\hline \multicolumn{10}{|l|}{ MSE for different sets } \\
\hline Train error & $3.3 E-05$ & $3.5 E-05$ & $1.4 E-05$ & $2.6 E-05$ & $2.4 E-05$ & $6.9 E-09$ & $7.0 E-09$ & $7.0 E-09$ & $6.8 E-09$ \\
\hline Validate error & 0.0005 & 0.0004 & 0.0005 & 0.0003 & 0.0005 & $3.0 E-06$ & $1.5 E-06$ & $2.0 E-06$ & $2.8 E-06$ \\
\hline Test error & 0.0008 & 0.0006 & 0.0007 & 0.0006 & 0.0005 & $4.4 E-06$ & $1.7 E-06$ & $4.7 E-06$ & $3.8 E-06$ \\
\hline \multicolumn{10}{|l|}{ For total data } \\
\hline MAE & 0.0111 & 0.0098 & 0.0088 & 0.0090 & 0.0089 & 0.0006 & 0.0004 & 0.0006 & 0.0005 \\
\hline MSE & 0.0003 & 0.0002 & 0.0002 & 0.0002 & 0.0002 & $1.5 E-06$ & $6.2 E-07$ & $1.3 E-06$ & $1.3 E-06$ \\
\hline RMSE & 0.0168 & 0.0151 & 0.0153 & 0.0142 & 0.0142 & 0.0012 & 0.0008 & 0.0012 & 0.0011 \\
\hline Minimum error & $8.5 E-06$ & $2.1 E-04$ & $2.6 E-05$ & $5.9 E-05$ & 0.0001 & $9.5 E-07$ & $1.7 E-07$ & $1.5 E-06$ & $6.5 E-08$ \\
\hline Maximum error & 0.0607 & 0.0677 & 0.0696 & 0.0701 & 0.0586 & 0.0059 & 0.0045 & 0.0060 & 0.0080 \\
\hline \multicolumn{10}{|c|}{ Relative error distribution } \\
\hline $0-1 \%$ & 152 & 157 & 152 & 157 & 155 & 162 & 162 & 162 & 162 \\
\hline $1 \%-5 \%$ & 10 & 5 & 10 & 5 & 7 & 0 & 0 & 0 & 0 \\
\hline $5 \%-10 \%$ & 0 & 0 & 0 & 0 & 0 & 0 & 0 & 0 & 0 \\
\hline $10 \%-50 \%$ & 0 & 0 & 0 & 0 & 0 & 0 & 0 & 0 & 0 \\
\hline$>50 \%$ & 0 & 0 & 0 & 0 & 0 & 0 & 0 & 0 & 0 \\
\hline
\end{tabular}

Network number 7 gives the best performance in the ANN models related to color-b prediction with the smallest MSE of 6.2E - 07.

TABLE 8: ANN statistical data for color-c prediction.

\begin{tabular}{lccccccccc}
\hline Network number & $\mathrm{N} 1$ & $\mathrm{~N} 2$ & $\mathrm{~N} 3$ & $\mathrm{~N} 4$ & $\mathrm{~N} 5$ & $\mathrm{~N} 6$ & $\mathrm{~N} 7$ & $\mathrm{~N} 8$ & $\mathrm{~N} 9$ \\
\hline MSE for different sets & & & & & & & & \\
$\quad$ Train error & $2.0 E-05$ & $3.6 E-06$ & $2.5 E-06$ & $3.6 E-06$ & $3.6 E-06$ & $4.1 E-09$ & $3.3 E-09$ & $4.8 E-09$ & $4.5 E-09$ \\
$\quad$ Validate error & $1.6 E-04$ & $1.6 E-04$ & 0.0002 & 0.0001 & $1.5 E-04$ & $1.5 E-07$ & $1.1 E-07$ & $2.2 E-07$ & $1.8 E-07$ \\
$\quad$ Test error & $2.1 E-04$ & 0.0001 & 0.0001 & 0.0001 & 0.0001 & $2.0 E-07$ & $9.8 E-08$ & $1.1 E-07$ & $2.6 E-07$ \\
For total data & & & & & & & & \\
MAE & 0.0062 & 0.0043 & 0.0048 & 0.0041 & 0.0041 & 0.0001 & 0.0001 & 0.0002 & 0.0002 \\
MSE & $8.6 E-05$ & $6.2 E-05$ & $7.6 E-05$ & $5.0 E-05$ & $5.4 E-05$ & $7.2 E-08$ & $\mathbf{4 . 3 E}-\mathbf{0 8}$ & $6.8 E-08$ & $9.3 E-08$ \\
RMSE & 0.0093 & 0.0079 & 0.0087 & 0.0070 & 0.0073 & 0.0003 & 0.0002 & 0.0003 & 0.0003 \\
Minimum error & $6.8 E-06$ & $2.5 E-06$ & $1.5 E-05$ & $3.5 E-05$ & $1.1 E-05$ & $2.8 E-06$ & $2.8 E-09$ & $5.5 E-07$ & $3.0 E-07$ \\
Maximum error & 0.0360 & 0.0382 & 0.0409 & 0.0320 & 0.0446 & 0.0016 & 0.0010 & 0.0012 & 0.0022 \\
Relative error distribution & & & & & & 162 & 162 & 162 \\
0-1\% & 162 & 162 & 161 & 162 & 161 & 162 & 162 \\
1\%-5\% & 0 & 0 & 1 & 0 & 1 & 0 & 0 & 0 \\
$5 \%-10 \%$ & 0 & 0 & 0 & 0 & 0 & 0 & 0 & 0 \\
$10 \%-50 \%$ & 0 & 0 & 0 & 0 & 0 & 0 & 0 & 0 \\
$>50 \%$ & 0 & 0 & 0 & 0 & 0 & 0 & 0 & 0 \\
\hline
\end{tabular}

Network number 7 gives the best performance in the ANN models related to color-c prediction with the smallest MSE of $4.3 E-08$.

error), and RMSE (rooted mean square error) are calculated. These three functions are widely used in evaluating the effect of fitting. Their definitions are as follows:

$$
\begin{gathered}
\mathrm{MAE}=\frac{1}{n} \sum_{i=1}^{n}\left(a b s\left(t_{i}-o_{i}\right)\right), \\
\mathrm{MSE}=\frac{1}{n} \sum_{i=1}^{n}\left(t_{i}-o_{i}\right)^{2}, \\
\mathrm{RMSE}=\sqrt{\mathrm{MSE}} .
\end{gathered}
$$

In the function, $t_{i}$ and $o_{i}$ are the target output and the ANN predicted output, respectively.
As mentioned before, the whole dataset were divided into training, validation, and test set. After training the networks, the models are expected to be used in large scale prediction works. They were validated by an unseen testing data set to predict the color values. Therefore, besides MAE and MSE, the test error, which is usually a symbol for the robustness and generalization of the model, is also an important factor in judging the quality of neural network.

Besides, some other statistical information was also collected. Detailed results for colour properties, that is, K/S Sum value (color-ks), CIE L* (color-l), CIE a* (color-a), CIE b* (color-b), CIE $\mathbf{C}^{*}$ (color-c) and $\mathrm{CIE} \mathbf{h}^{\circ}$ (color-h) values were shown in the Tables 4, 5, 6, 7, 8, and 9, respectively. Here, 
TABLE 9: ANN statistical data for color-h prediction.

\begin{tabular}{|c|c|c|c|c|c|c|c|c|c|}
\hline Network number & N1 & $\mathrm{N} 2$ & N3 & N4 & N5 & N6 & N7 & N8 & N9 \\
\hline \multicolumn{10}{|l|}{ MSE for different sets } \\
\hline Train error & $3.2 E-04$ & $7.7 E-05$ & $2.1 E-05$ & $7.1 E-05$ & $1.9 E-04$ & $4.3 E-06$ & $2.1 E-06$ & $1.5 E-06$ & $1.9 E-06$ \\
\hline Validate error & 0.0037 & 0.0030 & 0.0032 & 0.0013 & 0.0030 & $2.4 E-05$ & 0.0001 & 0.0001 & $9.1 E-05$ \\
\hline Test error & 0.0036 & 0.0018 & 0.0013 & 0.0042 & 0.0023 & $5.8 E-05$ & $4.8 E-05$ & $4.0 E-05$ & $7.4 E-05$ \\
\hline \multicolumn{10}{|l|}{ For total data } \\
\hline MAE & 0.0263 & 0.0185 & 0.0151 & 0.0181 & 0.0224 & 0.0028 & 0.0033 & 0.0033 & 0.0033 \\
\hline MSE & 0.0016 & 0.0010 & 0.0009 & 0.0011 & 0.0012 & $1.9 E-05$ & $3.3 E-05$ & $3.4 E-05$ & $3.4 E-05$ \\
\hline RMSE & 0.0402 & 0.0315 & 0.0300 & 0.0337 & 0.0341 & 0.0044 & 0.0058 & 0.0058 & 0.0058 \\
\hline Minimum error & $1.4 E-04$ & 0.0002 & $1.1 E-05$ & $8.1 E-06$ & 0.0002 & $1.1 E-06$ & $3.1 E-05$ & $6.3 E-06$ & $4.5 E-05$ \\
\hline Maximum error & 0.1859 & 0.1570 & 0.1982 & 0.2245 & 0.1437 & 0.0265 & 0.0279 & 0.0302 & 0.0316 \\
\hline \multicolumn{10}{|c|}{ Relative error distribution } \\
\hline $0-1 \%$ & 162 & 162 & 162 & 162 & 162 & 162 & 162 & 162 & 162 \\
\hline $1 \%-5 \%$ & 0 & 0 & 0 & 0 & 0 & 0 & 0 & 0 & 0 \\
\hline $5 \%-10 \%$ & 0 & 0 & 0 & 0 & 0 & 0 & 0 & 0 & 0 \\
\hline $10 \%-50 \%$ & 0 & 0 & 0 & 0 & 0 & 0 & 0 & 0 & 0 \\
\hline$>50 \%$ & 0 & 0 & 0 & 0 & 0 & 0 & 0 & 0 & 0 \\
\hline
\end{tabular}

Network number 6 gives the best performance in the ANN models related to color-h prediction with the smallest MSE of 1.9E - 05.

TABLE 10: Relative importance of the input variables.

\begin{tabular}{|c|c|c|c|c|c|}
\hline Excluded variable & Temperature & Time & $\mathrm{pH}$ & MA & Twist \\
\hline \multicolumn{6}{|c|}{ Color-ks (optimized MSE is 0.0015 ) } \\
\hline MSE & 1443.3 & 549.3 & 117.0 & 222.9 & 2630.4 \\
\hline$\%$ Increase & 96219900 & 36619900 & 7799900 & 14859900 & 175359900 \\
\hline \multicolumn{6}{|c|}{ Color-1 (optimized MSE is $9.9 E-06$ ) } \\
\hline MSE & 0.5835 & 0.2833 & 0.0629 & 0.0961 & 0.9708 \\
\hline$\%$ Increase & 5893839.4 & 2861516.2 & 635253.5 & 970607.1 & 9805960.6 \\
\hline \multicolumn{6}{|c|}{ Color-a (optimized MSE is $2.8 E-09$ ) } \\
\hline MSE & 0.0006 & 0.0003 & $1.2 E-05$ & 0.0001 & 0.0010 \\
\hline$\%$ Increase & 21428471.4 & 10714185.7 & 428471.4 & 3571328.6 & 35714185.7 \\
\hline \multicolumn{6}{|c|}{ Color-b (optimized MSE is $6.2 E-07$ ) } \\
\hline MSE & 0.0972 & 0.0625 & 0.0071 & 0.0099 & 0.1470 \\
\hline$\%$ Increase & 14507362.7 & 9328258.2 & 1059601.5 & 1477511.9 & 21940198.5 \\
\hline \multicolumn{6}{|c|}{ Color-c (optimized MSE is $4.3 E-08$ ) } \\
\hline MSE & 0.0557 & 0.0347 & 0.0024 & 0.0034 & 0.1007 \\
\hline$\%$ Increase & 129534783.7 & 80697574.1 & 5581295.3 & 7906876.7 & 234185946.5 \\
\hline \multicolumn{6}{|c|}{ Color-h (optimized MSE is $1.9 E-05$ ) } \\
\hline MSE & 8.3680 & 4.9060 & 0.2621 & 0.4304 & 9.7639 \\
\hline$\%$ Increase & 44042005.3 & 25820952.6 & 1379373.7 & 2265163.2 & 51388847.4 \\
\hline
\end{tabular}

for simplicity, only the network with the smallest MSE is highlighted as best network model.

It can be seen from Tables 4 to 9 that for two hidden layer neural networks, the train errors are normally smaller than one hidden layer network. However, it does not matter for the test error. On the other hand, the one layer network can also perform well in test error, which means it is more robust in most cases. From the Tables 4 to 9 , it can also note that in ANN model, the relative error distributes mostly in $0-5 \%$, which is an acceptable range.

3.2. The Relative Importance of the Input Variables. In order to find out the relative importance of these five input variables, an additional experiment was performed. In this experiment, all the input variables were remained except one designated. Then, the MAE, MSE, and other statistical information of the 
TABLE 11: LR model statistical data.

\begin{tabular}{|c|c|c|c|c|c|c|}
\hline & Color-ks & Color-1 & Color-a & Color-b & Color-c & Color-h \\
\hline MAE & 29.344 & 0.564 & 0.004 & 0.204 & 0.159 & 2.146 \\
\hline MSE & 1313.6 & 0.496 & $3.09 E-05$ & 0.079 & 0.051 & 8.195 \\
\hline RMSE & 36.244 & 0.704 & 0.006 & 0.281 & 0.227 & 2.863 \\
\hline Minimum error & 0.0211 & 0.0019 & $5.40 E-05$ & 0.0003 & 0.0001 & $3.40 E-04$ \\
\hline Maximum error & 86.687 & 1.946 & 0.0189 & 0.928 & 0.825 & 8.757 \\
\hline \multicolumn{7}{|c|}{ Relative error distribution } \\
\hline $0-1 \%$ & 21 & 29 & 159 & 26 & 32 & 120 \\
\hline $1 \%-5 \%$ & 67 & 101 & 3 & 76 & 91 & 42 \\
\hline $5 \%-10 \%$ & 60 & 27 & 0 & 33 & 28 & 0 \\
\hline $10 \%-50 \%$ & 14 & 5 & 0 & 27 & 11 & 0 \\
\hline$>50 \%$ & 0 & 0 & 0 & 0 & 0 & 0 \\
\hline
\end{tabular}

new ANN model were calculated and further compared with the optimized one. Here, for simplicity, the increase in the MSE value was treated as the indicator of the importance of the excluded input. The results are shown in Table 10.

It can be seen that the five input variables are all of great importance. Among them, fabric yarn twist level is the most significant one. This is because if all the process parameters are kept constant, the twist level in the yarn would affect the penetration of cellulase into the fabric to function the colour fading effect. A high level of twist in a yarn holds the fibres together and hence restricts the access of water to the yarn interior. Therefore, such a high yarn twist level would affect the cellulase absorption during the treatment. For other four variables in the process, treatment temperature plays a key role followed by treatment time, mechanical agitation, and $\mathrm{pH}$. Treatment temperature is the most important factor on cellulase (enzyme) treatment because enzyme reaction increases with temperature but it is only activated within a temperature range in which the enzyme structure maintains stable and unchanged. Beyond this optimum range, the enzyme activity will decrease sharply as the protein structure of enzyme is tangled through thermal agitation. Longer enzyme treatment time will prolong enzymatic degradation of cellulose and the time for further abrasion. With a longer treatment time, cellulase effectively hydrolyses fragments of cotton fibrils [16], and thus the fabric would be less fuzzy than the original [17]. Therefore, shorter treatment time with enzyme can preserve desired colour depth in denim fabrics. The level of mechanical action is altered by a varying number of steel balls applied in enzyme treatment. By increasing the number of steel balls applied, the abrasion of the fabric surface will increase which creates more accessible sites for enzyme attack. Denim fabric treated under no agitation has lighter shade than that treated with mild and vigorous agitation. It is because the cellulose fibres are weakened after treating with enzyme alone and are not well removed by mechanical agitation, [17]. The surface of fabric becomes more hairy and forming a layer of fuzz on surface [18]. For specimen treated under vigorous agitation, a biopolishing effect is obtained. The weaken fibres are well removed by strong mechanical agitation, and thus a cleaner and smoother surface is resulted which produce a lighter shade of fabric as dye particles are also removed with cellulose fibres. For specimen treated with mild agitation (50 steel balls), the situation is between zero agitation and vigorous agitation. Mild agitation is not strong enough to remove as many weaken surface fibres as vigorous agitation can, thus, rather, an uneven surface is produced under mild agitation. The uneven surface causes higher light diffusion on surface, and thus darker shade of specimen is resulted as they achieve lower reflectance on surface. Enzyme performs its maximal activity at a particular range of $\mathrm{pH}$ values. Deviation of the optimum $\mathrm{pH}$ range will alter the internal structure of enzyme due to the electrostatic interactions within the enzyme. Thus, the enzyme will no longer perform its normal function. As observed from Table 10, there is an increase in MSE when compared with the values in the optimized ANN model as shown in Tables 4 to 9. This means, if comparing them with normal models, removing some input variables may enhance the quality of ANN model. This is possible and reasonable because our experiment data is not sufficient enough. More variables in the input layer may cause the ANN to be overfitting.

3.3. Linear Regression (LR) Model. In this study, a traditional linear regression (LR) model was also established for the sake of comparison with ANN model. In general, response variable $y$ may be related to $k$ regressor variables. The function is shown in as follows:

$$
y=\beta_{0}+\beta_{1} x_{1}+\beta_{2} x_{2}+\cdots+\beta_{k} x_{k}+\varepsilon .
$$

The parameters $\beta_{i}$ are called the regression coefficients. This model describes a hyperplane in $k$-dimensional space of the regressor variables. The method of least square is typically used to estimate the regression coefficients in a multiple linear regression model. Here, the linear regression model is applied in aforementioned experiment data $[19,20]$. The results are shown in Table 11.

When comparing the ANN model and the LR model, it is evident that the predictive power of the ANN model is better than the LR model. From Table 11, all the evaluation numbers are obviously much greater than those of Tables 4 to 9 , which mean that applying LR model for prediction in the recent case 

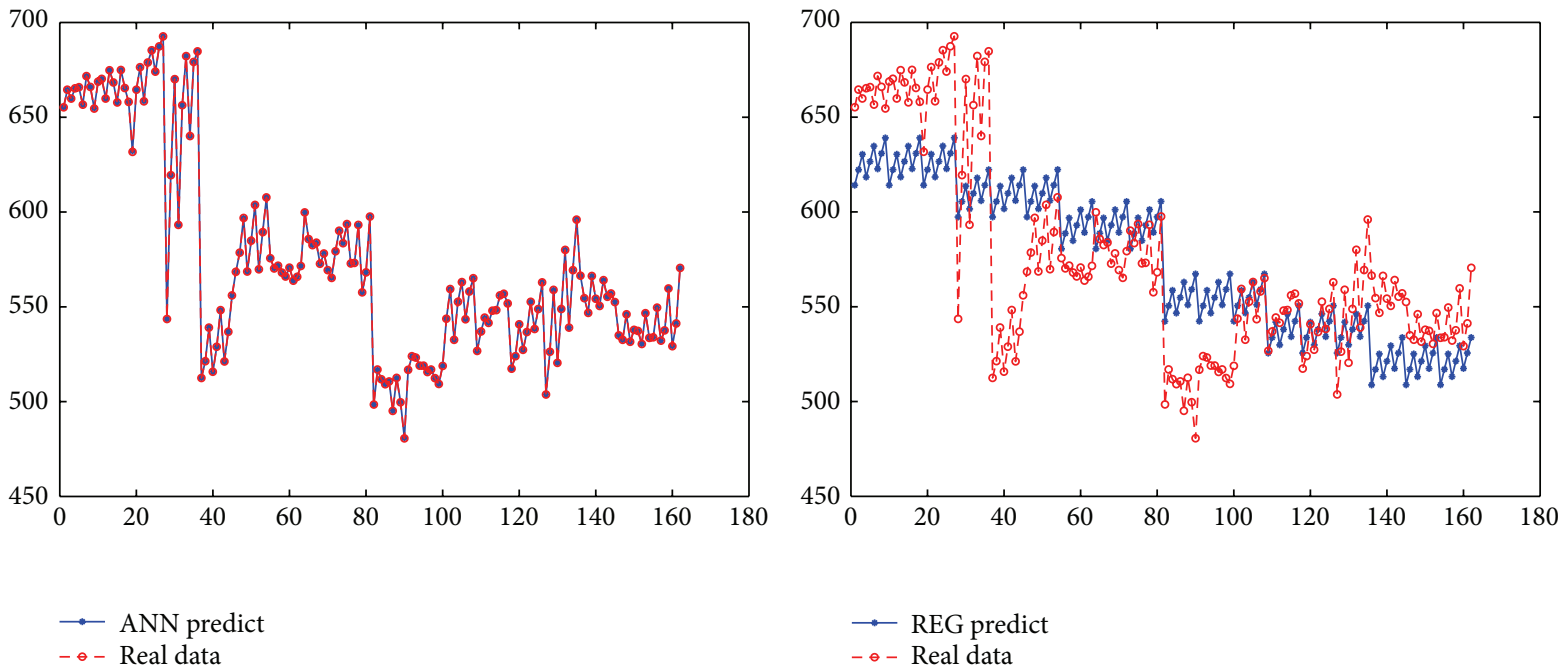

(a)

(b)

FIGURE 3: (a) ANN model fitting for color-ks. (b) LR model fitting for color-ks.
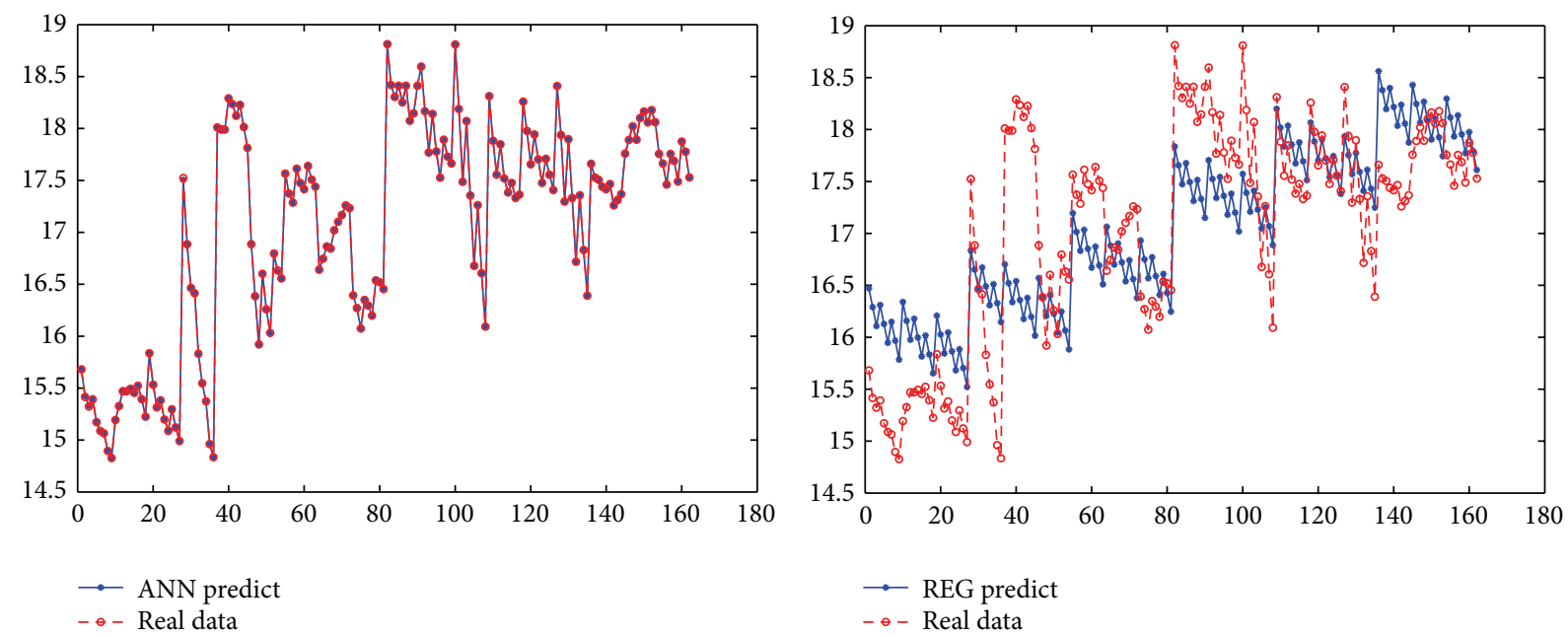

(a)

(b)

FIgURE 4: (a) ANN model fitting for color-l. (b) LR model fitting for color-l.

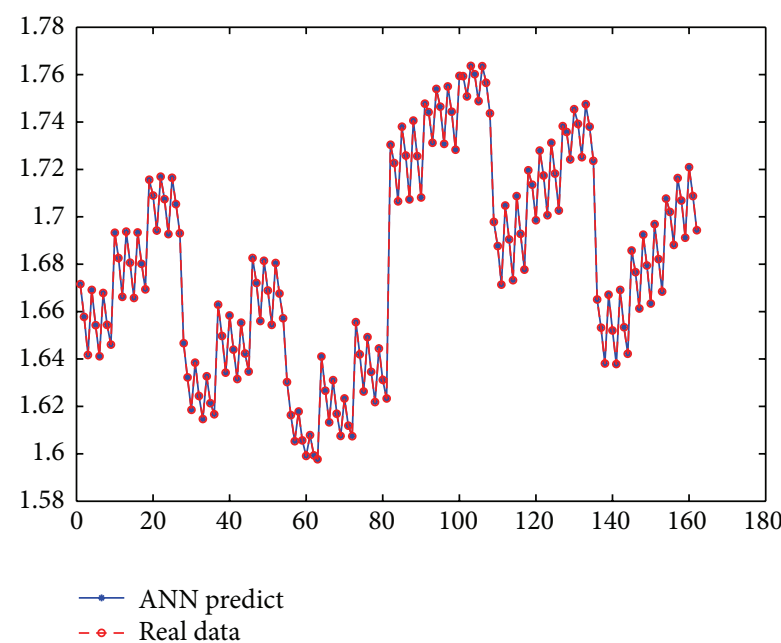

(a)

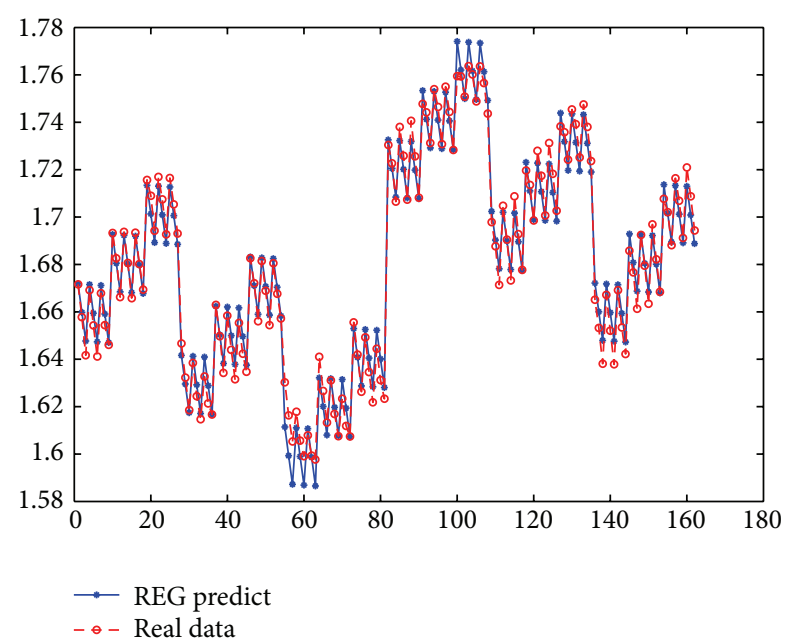

(b)

FIGURE 5: (a) ANN model fitting for color-a. (b) LR model fitting for color-a. 


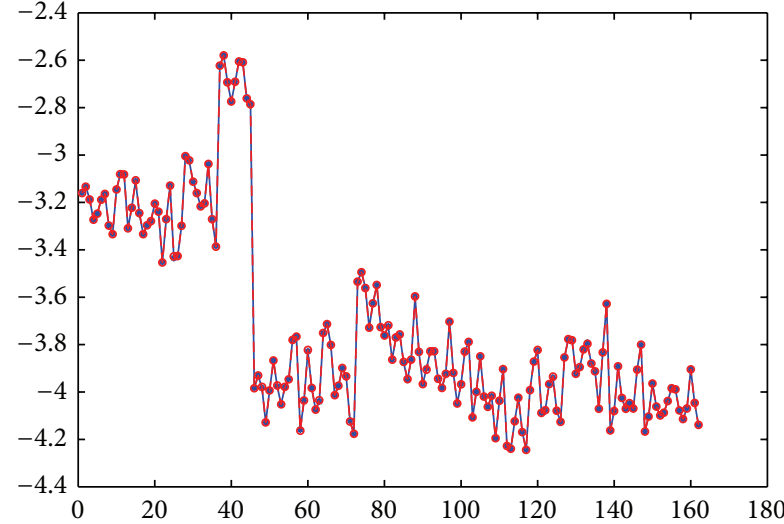

$\rightarrow$ ANN predict

$-\bullet$ Real data

(a)

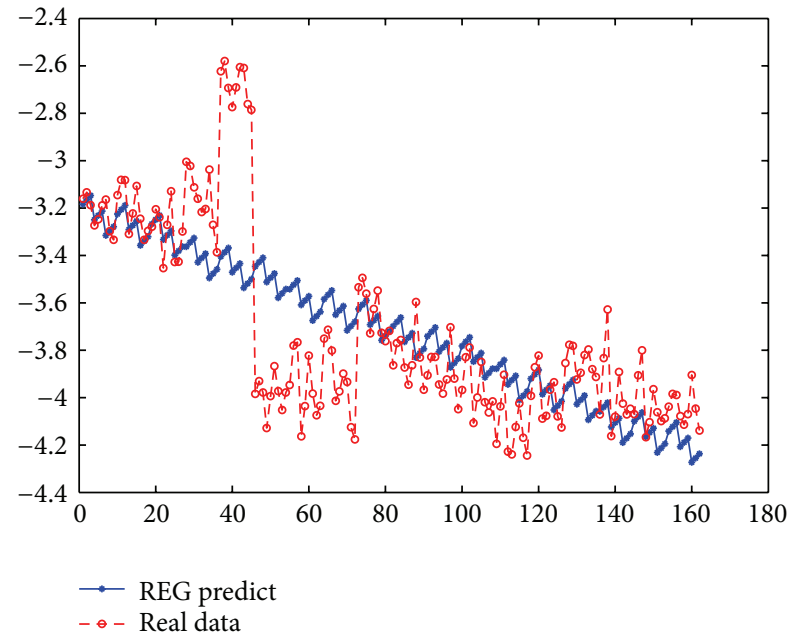

(b)

FIGURE 6: (a) ANN model fitting for color-b. (b) LR model fitting for color-b.
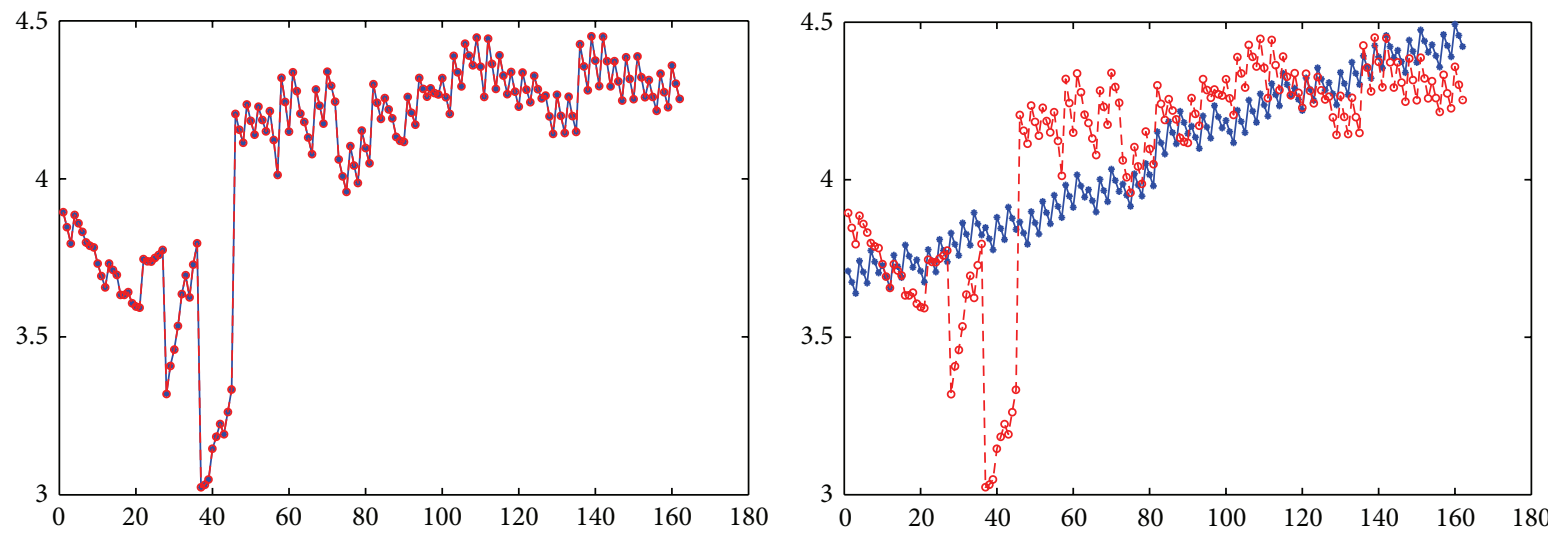

$\rightarrow$ ANN predict

$\rightarrow$ REG predict

$-\bullet$ - Real data

$-\theta-$ Real data

(a)

(b)

FIGURE 7: (a) ANN model fitting for color-c. (b) LR model fitting for color-c.
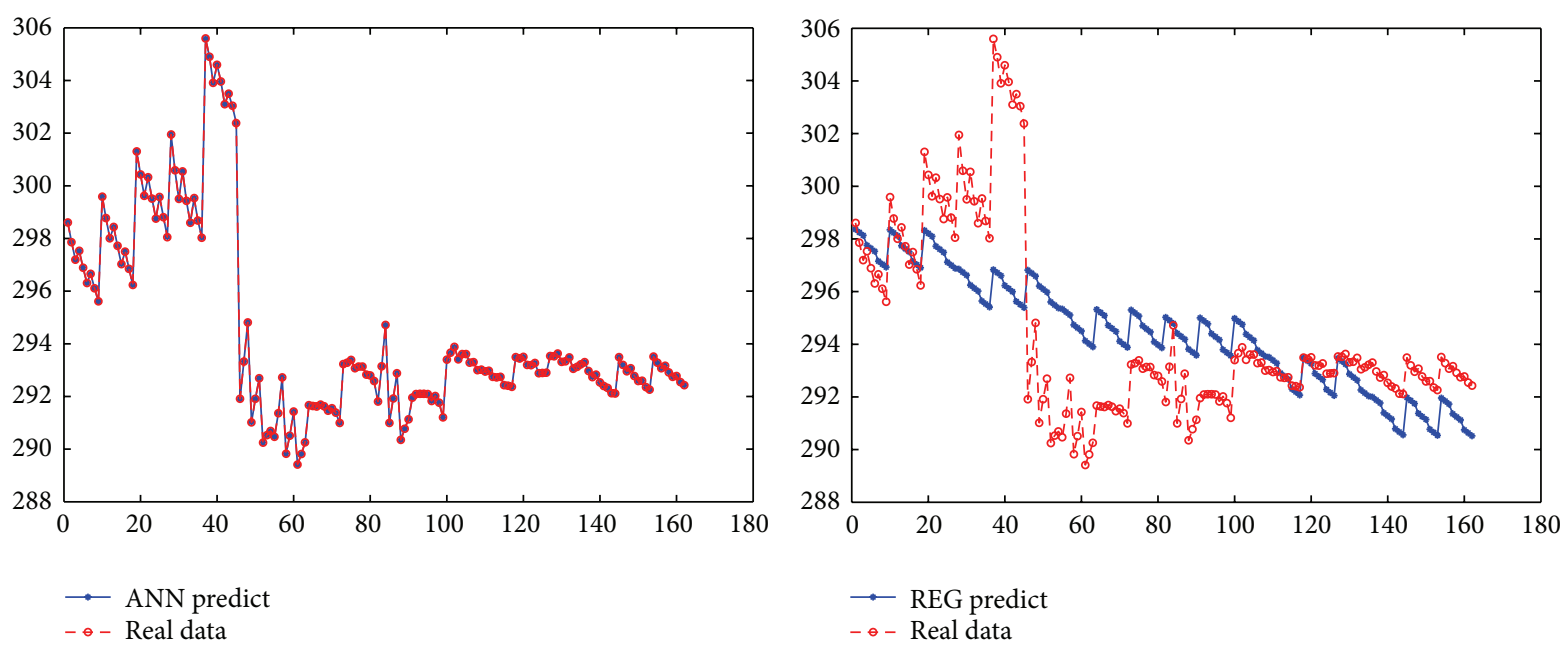

(a)

(b)

FIGURE 8: (a) ANN model fitting for color-h. (b) LR model fitting for color-h. 
is worse than ANN model. Figures 3, 4, 5, 6, 7 and 8 depict the comparison graphically.

\section{Conclusion}

In the paper, colour properties of $100 \%$ cotton denim fabrics, including colour yield (in terms of K/S value), CIE $\mathrm{L}^{*}, \mathrm{a}^{*}$, $\mathrm{b}^{*}, \mathbf{C}^{*}$, and $\mathbf{h}^{\circ}$ values, after cellulase enzyme treatment were predicted by using both ANN and LR models. Several statistical tests were conducted to examine the performance of these experiments and models. Experimental results revealed that changes in the number of nodes of the neural network model affected the performance of the model. Results also reflected that the colour properties could be predicted accurately with the help of the ANN model. These prediction results demonstrated the usefulness of cellulase treatment before coloring and might find good applications for future use by the textile industry. When a comparison was made between ANN and LR models, the LR model did not perform well in the experiment due to limitations of the model itself.

\section{Acknowledgment}

The Authors would like to acknowledge the financial support from The Hong Kong Polytechnic University for this work.

\section{References}

[1] Z. Gao, L. Zhang, and J. Zhao, "Application of laser technology in textile industry," Journal of Textile Research, vol. 27, no. 8, pp. 117-120, 2006.

[2] Z. Ondogan, O. Pamuk, E. N. Ondogan, and A. Ozguney, "Improving the appearance of all textile products from clothing to home textile using laser technology," Optics and Laser Technology, vol. 37, no. 8, pp. 631-637, 2005.

[3] L. C. Chang, F. J. Chang, and H. C. Hsu, "Real-time reservoir operation for flood control using artificial intelligent techniques," International Journal of Nonlinear Sciences and Numerical Simulation, vol. 11, no. 11, pp. 887-902, 2010.

[4] T. W. Lau, P. C. L. Hui, F. S. F. Ng, and K. C. C. Chan, "A new fuzzy approach to improve fashion product development," Computers in Industry, vol. 57, no. 1, pp. 82-92, 2006.

[5] L. Wu, K. L. Yick, S. P. Ng, J. Yip, and K. H. Kong, "Parametric design and process parameter optimization for bra cup molding via response surface methodology," Expert Systems With Applications, vol. 39, pp. 162-171, 2012.

[6] R. Beltran, L. Wang, and X. Wang, "Measuring the influence of fibre-to-fabric properties on the pilling of wool fabrics," Journal of the Textile Institute, vol. 97, no. 3, pp. 197-204, 2006.

[7] J. Fan and L. Hunter, "A worsted fabric expert system. II. An artificial neural network model for predicting the properties of worsted fabrics," Textile Research Journal, vol. 68, no. 10, pp. 763-771, 1998.

[8] P. K. Majumdar and A. Majumdar, "Predicting the breaking elongation of ring spun cotton yarns using mathematical, statistical, and artificial neural network models," Textile Research Journal, vol. 74, no. 7, pp. 652-655, 2004.

[9] C. M. Murrells, X. M. Tao, B. G. Xu, and K. P. S. Cheng, "An artificial neural network model for the prediction of spirality of fully relaxed single jersey fabrics," Textile Research Journal, vol. 79, no. 3, pp. 227-234, 2009.

[10] F. Pynckels, P. Kiekens, S. Sette, L. van Langenhove, and K. Impe, "Use of neural nets for determining the spinnability of fibres," Journal of the Textile Institute, vol. 86, no. 3, pp. 425-437, 1995.

[11] K. Hornik and M. Stinchombe, Multilayer Feed-Forward Networks Are Universal Approximators in Artificial Neural Networks: Approximation and Learning Theory, Blackwell Press, Oxford, UK, 1992.

[12] R. Hetcht-Nielsen, "Theory of the backpropagation neural networks," in Proceedings of International Joint Conference on Neural Networks (IJCNN '89), vol. 1, pp. 593-611, 1989.

[13] G. Cybenko, "Approximation by superpositions of a sigmoidal function," Mathematics of Control, Signals, and Systems, vol. 2, no. 4, pp. 303-314, 1989.

[14] S. C. Huang and Y. F. Huang, "Bounds on the number of hidden neurons in multilayer perceptrons," IEEE Transactions on Neural Networks, vol. 2, no. 1, pp. 47-55, 1991.

[15] M. A. Sartori and P. J. Antsaklis, "A simple method to derive bounds on the size and to train multilayer neural networks," IEEE Transactions on Neural Networks, vol. 2, no. 4, pp. 467471, 1991.

[16] A. S. Aly, A. B. Moustafa, and A. Hebeish, "Bio-technological treatment of cellulosic textiles," Journal of Cleaner Production, vol. 12, no. 7, pp. 697-705, 2004.

[17] C. W. Kan, C. W. M. Yuen, and S. Q. Jiang, "The effect of cellulase treatment on hydrolysis of linen," Fibers and Polymers, vol. 7, no. 3, pp. 241-244, 2006.

[18] A. Cavaco-Paulo, "Mechanism of cellulase action in textile processes," Carbohydrate Polymers, vol. 37, no. 3, pp. 273-277, 1998.

[19] O. N. Hung, L. J. Song, C. K. Chan, C. W. Kan, and C. W. M. Yuen, "Using artificial neural network to predict color properties of laser-treated 100\% cotton fabric," Fibers and Polymers, vol. 12, pp. 1069-1076, 2011.

[20] O. N. Hung, L. J. Song, C. K. Chan, C. W. Kan, and C. W. M. Yuen, "Predicting the laser-engraved color properties on cotton-spandex fabric by artificial neural network," AATCC Review, vol. 12, no. 3, pp. 57-63, 2012. 

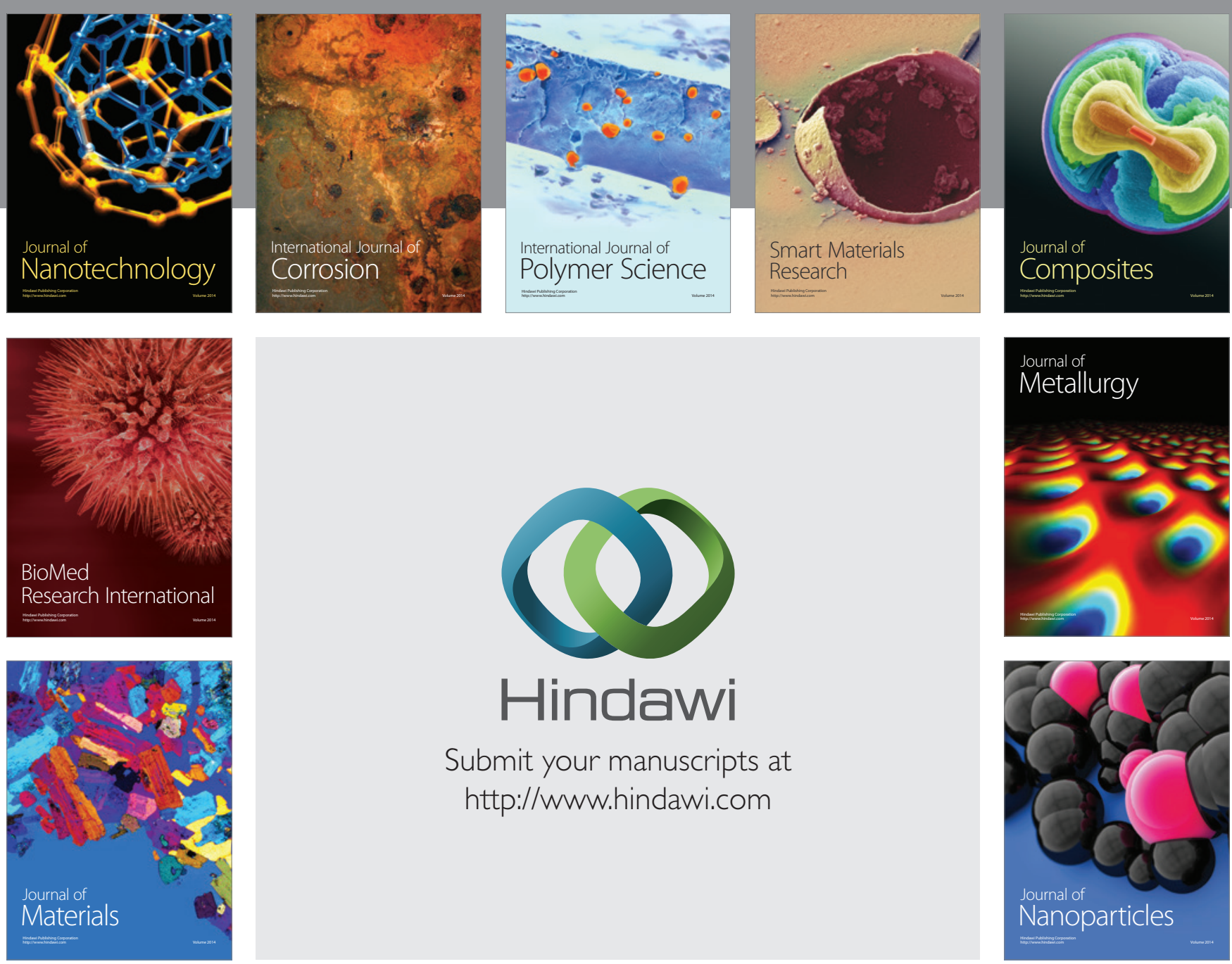

Submit your manuscripts at http://www.hindawi.com
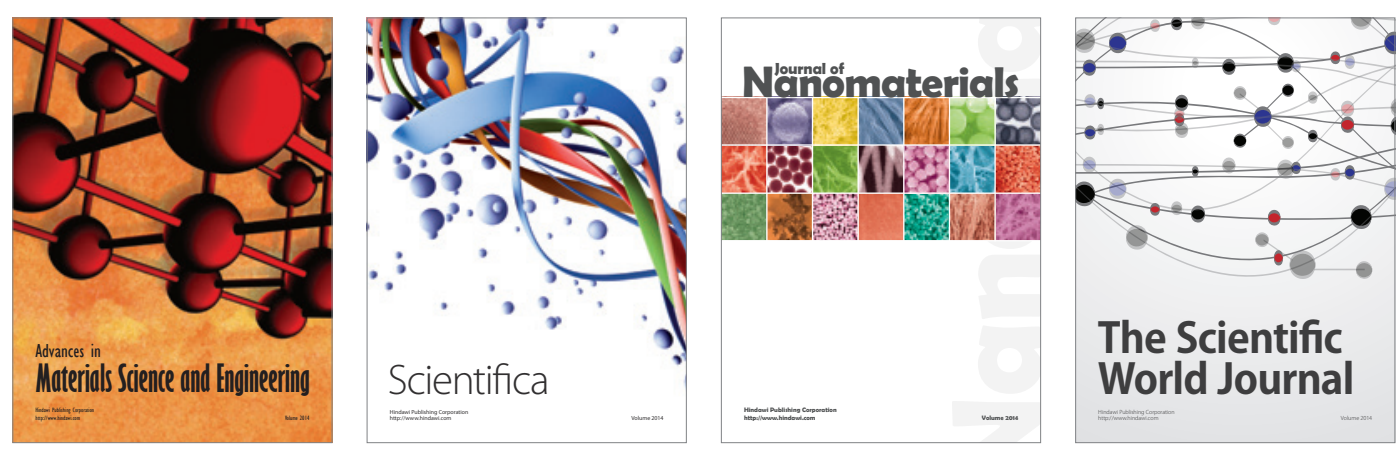

\section{The Scientific World Journal}
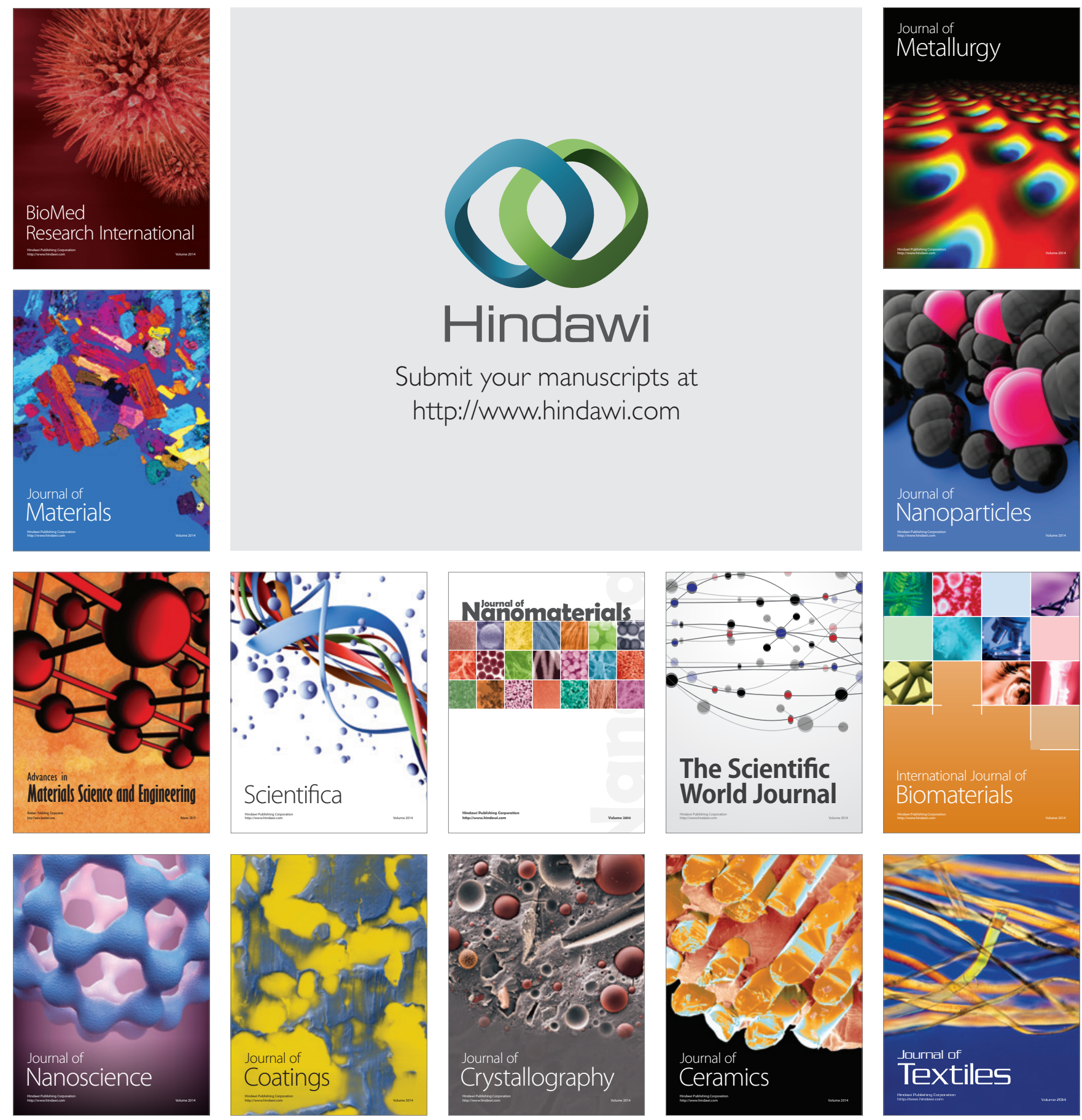